\title{
Globe
}

Revue internationale d'études québécoises

\section{Jean Crête [éd.] : Politiques publiques : le Québec comparé, Québec, Presses de l’Université Laval, 2006}

\section{Peter Graefe}

Volume 10, numéro 1, 2007

URI : https://id.erudit.org/iderudit/1000120ar

DOI : https://doi.org/10.7202/1000120ar

Aller au sommaire du numéro

Éditeur(s)

Globe, Revue internationale d'études québécoises

ISSN

1481-5869 (imprimé)

1923-8231 (numérique)

Découvrir la revue

Citer ce compte rendu

Graefe, P. (2007). Compte rendu de [Jean Crête [éd.] : Politiques publiques : le

Québec comparé, Québec, Presses de l'Université Laval, 2006]. Globe, 10(1),

186-188. https://doi.org/10.7202/1000120ar d'utilisation que vous pouvez consulter en ligne.

https://apropos.erudit.org/fr/usagers/politique-dutilisation/ 
terminologiques auraient été les bienvenues ainsi qu'une division du matériel, afin de mieux servir la particularité de chaque discours, approche ou discipline et une conclusion, pour résumer la contribution des analyses à la compréhension des liens entre discours et identité. Nonobstant ces quelques réserves, je tiens à saluer les analyses menées, surtout par les étudiants, pour l'intérêt de leurs sujets ainsi que pour la qualité de l'écriture, de la documentation, de la structure et de l'argumentation.

Katri Suhonen

Université Concordia

\section{Jean Crête [éd.]}

Politiques publiques: le Québec comparé

Québec, Presses de l'Université Laval, 2006.

Ce livre, issu d'un colloque qui a eu lieu à l'Université Laval en 2002, déclare l'intention de parler du Québec, "d'un Québec mis en contexte, d'un Québec comparé." Je ne peux que saluer cette volonté de comprendre le Québec, vu ici sous l'angle de ses choix de politiques publiques, dans le contexte de l'expérience d'autres sociétés. Il y a en effet tout un corpus universitaire sur l'exceptionnalisme québécois, par exemple sur un modèle socio-économique nommé Québec Inc. ou modèle québécois, qui serait unique en Amérique du Nord. On entend souvent dans les médias et sur les tribunes politiques que le Québec aurait une culture plus socialdémocrate et plus portée à la concertation que celle de ses voisins. Il y a sans doute du bien-fondé dans ces affirmations, mais il nous manque toujours les études comparatives pour ajouter les nuances nécessaires et démontrer les limites de cet exceptionnalisme. Mais malgré la nécessité de poursuivre une démarche comparative, ce collectif n'augmente pas notre compréhension du Québec de manière importante. En effet, elle montre tous les défauts typiques d'un recueil de textes tirés d'un colloque : il y a plusieurs contributions intéressantes, mais la qualité et la pertinence des textes sont inégales, et la somme est moindre que les parties.

L'introduction essaie de situer la problématique du collectif autour de l'importance de la méthode comparative, quoique l'espace accordé à cette question (environ 6 pages) soit nettement insuffisant, laissant de côté plusieurs questions épistémologiques et méthodologiques courantes en ce qui a trait à la recherche sur les méthodes dans les domaines de la politique 
comparée et des politiques publiques comparées. Le directeur de l'ouvrage aurait dû se permettre plus de pages pour présenter cette question. En plus, l'accent méthodologique de l'introduction semble signaler que les auteurs n'avaient pas rédigé leurs textes autour d'une problématique serrée, et cela se vérifie à la lecture de ces derniers. En effet, plusieurs textes restent dans un registre descriptif, se contentant de décrire les politiques québécoises et leurs ressemblances avec les politiques publiques d'ailleurs. On pense ici aux textes de Dominique Daniel sur la politique d'immigration, de Gilbert Charland sur les finances publiques, de Lise Dubois et Aleck Ostry sur la politique d'alimentation et de Lise Lamothe sur le contrôle des médicaments. D'autres textes, comme ceux d'Éric Montpetit sur la procréation médicalement assistée, de Louis Imbeau et François Pétry sur la politique budgétaire, et de Jean Mercier et ses co-auteurs sur la gestion des matières résiduelles, prennent un ton plus analytique, cherchant à expliquer pourquoi les politiques québécoises étaient similaires aux politiques d'ailleurs ou en différaient. Enfin, deux textes, par ailleurs fort intéressants, n'abordent la question des politiques publiques que dans le contexte de "quoi faire" face aux défis des changements démographiques (Jacques Palard) et de la faible compétence civique des Québécois (Henry Milner).

On a donc raison de se questionner sur l'utilité de cette publication. Pour les chercheurs dans le domaine des politiques publiques et de la politique québécoise, il y a des observations pointues et des données comparatives qui pourraient être utiles, mais pas de grandes surprises ou d'innovations. Les spécialistes de certains champs de politiques, comme la politique budgétaire, la procréation médicalement assistée, l'utilisation de la recherche universitaire par la fonction publique ou la gestion des matières résiduelles, trouveront des arguments intéressants et intelligents, quoique les auteurs de ces textes aient pour la plupart déjà publié les résultats de leurs recherches ailleurs. Par contre, pour les québécistes cherchant une introduction aux politiques publiques québécoises, ce collectif aura une utilité limitée. Bien que quelques chapitres déjà mentionnés donnent un aperçu de questions ayant un intérêt transdisciplinaire (immigration, démographie), plusieurs chapitres abordent des politiques moins connues et moins centrales dans les grandes missions de l'État, comme la procréation médicalement assistée, la gestion des matières résiduelles et la politique alimentaire. Bref, ce collectif ne peut pas servir d'introduction aux politiques publiques québécoises au sens large, quoiqu'il faille souligner que le directeur ne cherchait pas à élaborer un manuel. Compte tenu de l'absence d'une 
problématique plus serrée, l'ouvrage ne réussit pas non plus à développer des thèmes plus larges (l'exceptionnalisme québécois, l'impact de la mondialisation ou du néolibéralisme sur les politiques publiques), qui auraient pu alimenter des débats transdisciplinaires sur le Québec.

Bref, malgré son intention de mettre le Québec en contexte, le collectif ne réussit pas à fournir un cadre théorique unifié qui justifierait la comparaison. Le choix de la comparaison en est un surtout méthodologique, motivé par sa capacité à approfondir une problématique ou une question de recherche précise. Le résultat, dans le cas de ce livre, est une série de chapitres aux problématiques fort différentes, qui avancent très peu notre appréciation de la place du Québec dans ses contextes géographiques (continental, mondial) ou temporels (modernité, postmodernité, capitalisme post-industriel, etc.).

Peter Graefe

Université de McMaster

\section{Annette Hayward \\ La querelle du régionalisme au Québec (1904-1931). Vers l'autonomisation de la littérature québécoise Ottawa, Le Nordir, 2006.}

Cette publication recèle un paradoxe, propre à désarçonner la critique. En 1980, Annette Hayward soutenait une thèse de plus de 1000 pages, intitulée "Le conflit entre les régionalistes et les exoriques au Québec (1900-1920)"; depuis, bien peu de publications consacrées au régionalisme, au Nigog ou aux premières décennies du XX $\mathrm{XX}^{e}$ siècle ne s'y réfèrent pas. Désormais, les chercheurs devront refaire leurs notes, pour référer au livre. Ils n'auront cependant pas à le relire, puisqu'à l'exception de très légers retranchements et de retouches stylistiques mineures, rien n'a été changé au texte initial.

Cette parution d'un texte dont la fortune critique est un fait avéré, lui-même susceptible d'une étude historique, constitue à toutes fins pratiques une réédition. Il nous parvient d'ailleurs pourvu d'un discours d'escorte caractéristique: une préface de Dominique Garand et une brochure publicitaire rassemblant les éloges de Marie-Andrée Beaudet, Jacques Michon et François Ricard. Bref, voilà un classique de la critique littéraire historique, désormais plus accessible. 